\title{
Bi-stable dynamics of a host-pathogen model
}

\author{
Roumen Anguelov* ${ }^{* \dagger}$, Rebecca Bekker*, Yves Dumont $* \ddagger$ \\ * Department of Mathematics and Applied Mathematics, University of Pretoria, South Africa \\ roumen.anguelov@up.ac.za, rebeccaabekker@gmail.com \\ $\dagger$ Associate Member of the Institute of Mathematics and Informatics \\ Bulgarian Academy of Sciences, Sofia , Bulgaria \\ $\ddagger$ CIRAD, Umr AMAP, Pretoria, South Africa \\ AMAP, University of Montpellier, CIRAD, CNRS, INRA, IRD, Montpellier, France \\ yves.dumont@cirad.fr
}

Received: 8 August 2018, accepted: 2 January 2019, published: 23 January 2019

\begin{abstract}
Crop host-pathogen interaction have been a main issue for decades, in particular for food security. In this paper, we focus on the modeling and long term behavior of soil-borne pathogens. We first develop a new compartmental temporal model, which exhibits bi-stable asymptotical dynamics. To investigate the long term behavior, we use LaSalle Invariance Principle to derive sufficient conditions for global asymptotic stability of the pathogen free equilibrium and monotone dynamical systems theory to provide sufficient conditions for permanence of the system. Finally, we develop a partially degenerate reaction diffusion system, providing a numerical exploration based on the results obtained for the temporal system. We show that a traveling wave solution may exist where the speed of the wave follows a power law.
\end{abstract}

Keywords-Host-pathogen; bi-stability; monotone dynamical system; LaSalle Invariance Principle; partially degenerate reaction-diffusion; traveling wave

\section{INTRODUCTION}

The global food supply is currently experiencing pressure from climate change and ever increasing demand. Another major concern is the increasing impact of pathogens. An estimated $16 \%$ of the global crop yield is lost to various pathogens annually [3], [10], [14]. Consequently there has been an increase in research of botanical pathogens and the resulting diseases, with foliar pathogens being the focus of the majority of published work. One important difference between foliar and soil-borne pathogens is the environment wherein each occurs. Foliar pathogens have to contend with external factors such as wind, radiation and varying temperatures. However, the soil environment dampens the effects of such factors, although the inherent opacity of soil poses a number of challenges of its own. Added to these are challenges relating to capturing the direct and indirect influence of the environment on processes such as survival,

Copyright: (c) 2019 Anguelov et al. This article is distributed under the terms of the Creative Commons Attribution License (CC BY 4.0), which permits unrestricted use, distribution, and reproduction in any medium, provided the original author and source are credited.

Citation: Roumen Anguelov, Rebecca Bekker, Yves Dumont, Bi-stable dynamics of a host-pathogen model, Biomath 8 (2019), 1901029, http://dx.doi.org/10.11145/j.biomath.2019.01.029 
dispersal and germination of pathogens, tissue growth, spatial distribution and susceptibility of hosts [12].

To some extent this paper is motivated by an early work of Gilligan [6], [7], [8], where he proposed a SEIR type compartmental model for the propagation of a soil-borne plant disease. This model includes a diffusion term, suggesting movement of infectious individuals. Although correct in certain contexts, this is unlikely in plant populations. In the model proposed here we consider compartments for the pathogen, where infection/infestation occurs when pathogen attaches to susceptible roots. In the spatio-temporal model we consider diffusion of the unattached pathogen, which we believe is a more biologically realistic assumption.

The paper is organized as follows. In the next section we construct the temporal host-pathogen model highlighting the assumptions on which it is based. Section 3 deals with the equilibria of the system. Sufficient conditions for extinction and persistence of the pathogen are presented in Section 4 and 5 respectively. The spatio-temporal model is numerically considered in Section 6.

\section{THE HOST-PATHOGEN MODEL}

We consider a population of susceptible host plants with a constant recruitment rate $\Lambda$, and a pathogen present in the soil. As usual, the compartments of susceptible and infective/infested hosts are denoted by $S$ and $I$, respectively, and $N=S+I$ is the total host population. The natural decay rate of the host is $d$ per time unit, and infected hosts have an addition decay rate of $\alpha$ per time unit. We assume that the pathogen is dependent on its host for nutrients or energy, and as such has an expected off-host death rate $\delta$ per time units. After coming into contact with a susceptible host it attaches at rate $\rho$, and grows at intrinsic growth rate of $\lambda$, restricted by the carrying capacity $\gamma I$ of the infected/infested roots. The attached pathogen (compartment $A$ ) detaches from their hosts at a rate of $\sigma$ per time unit. The unattached or free pathogen (compartment $F$ ) is responsible for new infections/infestations. It is assumed that if the population of free pathogen is large, the transmission rate from $S$ to $I$ depends solely on a constant $\beta$ and the level of susceptible hosts present. This type of incidence is called saturation incidence and we use the specific form $\frac{\beta F}{M+F}$. Using a saturating infestation rate is motivated by biological observations that increasing the free pathogen beyond a certain level no longer increases infestation proportionally. From a mathematical point of view, if only mass action principle is applied e.g. $\beta F S$, then, since $\mathrm{F}$ can potentially be very large, $S$ would decrease very rapidly, which is unrealistic. For simplicity, the attachment rate (transfer from $F$ to $A$ is just mass action principle, namely $\rho F S$. However, the growth in the $A$ compartment is limited through the carrying capacity $\gamma I=\gamma(N-S)$. Since $S$ cannot decrease unrealistically quickly then $A$ cannot increase unrealistically quickly. The flow

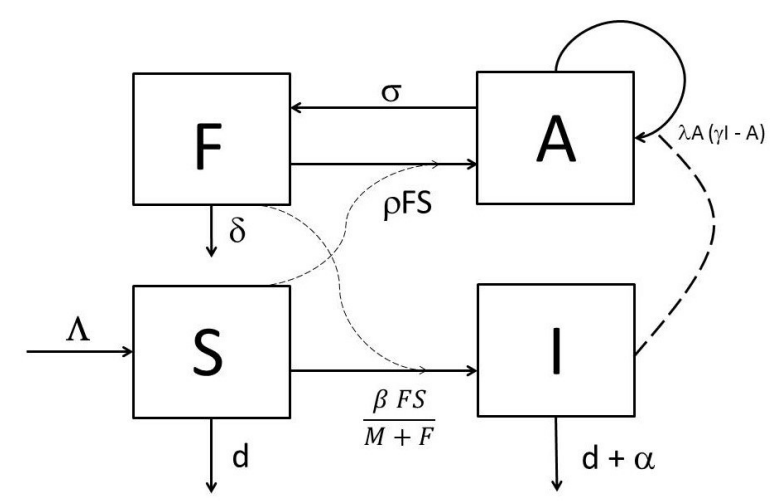

Fig. 1. Flow chart of the host-pathogen model

chart is given in Figure 1. The model is a system of ODEs presented below:

$$
\begin{aligned}
\frac{d A}{d t} & =\lambda A(\gamma I-A)-\sigma A+\rho F S \\
\frac{d F}{d t} & =-\delta F+\sigma A-\rho F S \\
\frac{d S}{d t} & =\Lambda-d S-\frac{\beta F}{M+F} S \\
\frac{d I}{d t} & =\frac{\beta F}{M+F} S-(\alpha+d) I
\end{aligned}
$$

Using the notation $x=(A, F, S, I)^{T}$ the model 
(1) is written as

$$
\dot{x}=f(x)
$$

with

$$
f(x)=\left(\begin{array}{c}
\lambda A(\gamma I-A)-\sigma A+\rho F S \\
-\delta F+\sigma A-\rho F S \\
\Lambda-d S-\frac{\beta F}{M+F} S \\
\frac{\beta F}{M+F} S-(\alpha+d) I
\end{array}\right) .
$$

The local existence and uniqueness of solutions of $\sqrt{1}$ in $\mathbb{R}_{+}^{4}=\left\{x \in \mathbb{R}^{4}: x \geq 0\right\}$ follows from the fact that $f$ is continuously differentiable on $\mathbb{R}_{+}^{4}$. The vector field defined by $f$ points inwards on the boundary of $\mathbb{R}_{+}^{4}$. Hence, $\mathbb{R}_{+}^{4}$ is positively invariant. In order to obtain global existence of solutions it remains to show that all solutions initiated in $\mathbb{R}_{+}^{4}$ are bounded. Adding the equations for $\frac{d S}{d t}$ and $\frac{d I}{d t}$ we have the inequalities

$$
\Lambda-(d+\alpha)(S+I) \leq \frac{d(S+I)}{d t} \leq \Lambda-d(S+I),
$$

which do not depend on the other coordinates of $x$. Hence, for any solution we have that the interval $\left[\frac{\Lambda}{\alpha+d}, \frac{\Lambda}{d}\right]$ is a global attractor for $S(t)+I(t)$. More precisely, we have

$$
\begin{aligned}
\frac{\Lambda}{\alpha+d} & \leq \liminf _{t \rightarrow+\infty}(S(t)+I(t)) \\
& \leq \limsup _{t \rightarrow+\infty}(S(t)+I(t)) \leq \frac{\Lambda}{d}
\end{aligned}
$$

Since $S$ and $I$ are also nonnegative, they are bounded. Using (4) it is easy to obtain that the rest of the coordinates of any solution $x(t)$ are also bounded. In fact, since the bounds in (4) do not depend on the initial condition, one obtains that $(1)$ defines a dynamical system on $\mathbb{R}_{+}^{4}$, which is dissipative.

\section{EQUILIBRIA}

In the absence of pathogen the population of the host is $S$ and it is governed by the third equation in the model (1). It has an asymptotically stable equilibrium at $\frac{\Lambda}{d}$ with basin of attraction $S \in[0,+\infty)$.
The resulting equilibrium of the model (1) we call Pathogen Free Equilibrium (PFE), that is,

$$
\mathbf{P F E}=\left(0,0, \frac{\Lambda}{d}, 0\right) .
$$

The basic reproduction number/ratio, $\mathcal{R}_{0}$, is a threshold quantity, which is often used to characterize the properties of epidemiological models. It is popularly defined as the number of new infections caused by a single infectious individual in a wholly susceptible population. Its precise definition is that it is the spectral radius of the next generation matrix calculated at an asymptotically stable equilibrium of the population in the absence of disease, [17]. Such equilibrium is usually referred to as Disease Free Equilibrium. Due to the nature of the model in this paper, and as mentioned above, we use the term Pathogen Free Equilibrium (PFE). The model (1) has a unique Pathogen Free Equilibrium given by (5).

Following the method in [17] for the computation of $\mathcal{R}_{0}$, the compartment vector $x$ in the model (1) is decomposed into three-dimensional vector of pathogen related compartments $y=$ $(A, F, I)^{T}$ and one pathogen free compartment $S$ and we have

$$
\dot{y}=\mathcal{F}(y, S)-\mathcal{V}(y, S),
$$

where

$$
\begin{aligned}
& \mathcal{F}(y, S)=\left(\begin{array}{c}
\lambda A(\gamma I-A)+\rho S F \\
0 \\
\frac{\beta F S}{M+F}
\end{array}\right), \\
& \mathcal{V}(y, S)=\left(\begin{array}{c}
\sigma A \\
\delta F-\sigma A+\rho F S \\
(\alpha+d) I
\end{array}\right) .
\end{aligned}
$$

The next generation matrix is

$$
\begin{aligned}
& \frac{\partial \mathcal{F}}{\partial y}\left(0, \frac{\Lambda}{d}\right)\left(\frac{\partial \mathcal{V}}{\partial y}\left(0, \frac{\Lambda}{d}\right)\right)^{-1} \\
& \quad=\frac{1}{M\left(\delta+\rho \frac{\Lambda}{d}\right)}\left(\begin{array}{ccc}
\rho M \frac{\Lambda}{d} & \rho M \frac{\Lambda}{d} & 0 \\
0 & 0 & 0 \\
\beta \frac{\Lambda}{d} & \beta \frac{\Lambda}{d} & 0
\end{array}\right) .
\end{aligned}
$$

Thus

$$
\mathcal{R}_{0}=\frac{\rho \Lambda}{d \delta+\rho \Lambda} .
$$


It is clear that $\mathcal{R}_{0}<1$ regardless of the values of the parameters (as long as they are positive). Therefore, it follows from [17, Theorem 2] that the PFE is always asymptotically stable. The asymptotic behavior of the solutions of (1) depends on the existence of pathogen-endemic equilibria or other invariant sets and their properties. It is interesting to remark that for the model under consideration $\mathcal{R}_{0}$ is not a threshold quantity at all.

In order to obtain all equilibria, we set right hand side in model (1) equal to zero,

$$
\begin{aligned}
\lambda A(\gamma I-A)-\sigma A+\rho F S & =0, \\
-\delta F+\sigma A-\rho F S & =0, \\
\Lambda-d S-\frac{\beta F}{M+F} S & =0, \\
\frac{\beta F}{M+F} S-(\alpha+d) I & =0 .
\end{aligned}
$$

From the third equation in (6) we obtain an expression for $S$ in terms of $F$ :

$$
S=\frac{\Lambda(M+F)}{(d+\beta) F+d M} .
$$

Then, using (7), from the second equation in (6) we obtain an expression for $A$ :

$$
\begin{aligned}
A & =\sigma^{-1}(\delta+\rho S) F \\
& =\sigma^{-1}\left(\delta+\frac{\rho \Lambda(M+F)}{(d+\beta) F+d M}\right) F .
\end{aligned}
$$

Similarly, using (7), the fourth equation in (6) gives an expression for $I$ in terms of $F$ :

$$
\begin{aligned}
I & =\frac{\beta F S}{(\alpha+d)(M+F)} \\
& =\frac{\Lambda \beta F}{(\alpha+d)((d+\beta) F+d M)} .
\end{aligned}
$$

Substituting these expressions into the first equation and excluding the case $F=0$, we obtain a cubic equation about $F$ in the form

$$
-a_{1} F^{3}+a_{2} F^{2}+a_{3} F-a_{4}=0
$$

where

$$
\begin{aligned}
a_{1}= & \lambda(\alpha+d)[\delta(d+\beta)+\rho \Lambda]^{2} \\
a_{2}= & -2 \lambda(\alpha+d)[\delta(d+\beta)+\rho \Lambda](\delta d+\rho \Lambda) M \\
& -\delta(\alpha+d)(d+\beta)^{2} \sigma^{2} \\
& +\lambda \gamma \Lambda \sigma \beta[\delta(d+\beta)+\rho \Lambda] \\
a_{3}= & \lambda \gamma \sigma \Lambda \beta(\delta d+\rho \Lambda) M-2 \delta(\alpha+d)(d+\beta) d M \sigma^{2} \\
& -\lambda(\alpha+d)(\delta d+\rho \Lambda)^{2} M^{2} \\
a_{4}= & \delta(\alpha+d) \sigma^{2} d^{2} M^{2}
\end{aligned}
$$

Clearly, $a_{1}>0$ and $a_{4}>0$, while the signs of $a_{2}$ and $a_{3}$ may vary depending on the values of the parameters. However, it is easy to see that for any signs of $a_{2}$ and $a_{3}$ there are always either two sign changes in the sequence of the coefficients of (10) or no sign changes at all. Hence the equation has either two positive roots or no positive roots. When these roots exist we denote them by $F_{1}$ and $F_{2}$, with $F_{1} \leq F_{2}$. The respective equilibria of the model 10 are denoted by $\mathcal{E} \mathcal{E}_{1}=$ $\left(A_{1}, F_{1}, S_{1}, I_{1}\right)^{T}$ and $\mathcal{E}_{2}=\left(A_{2}, F_{2}, S_{2}, I_{2}\right)^{T}$. From the expressions (7), (8) and (9) we see that $\mathcal{E E}_{1}>0$ and $\mathcal{E E}_{2}>0$. Further, we can also see from the expressions (7)-(9) that $S$ is a decreasing function of $F$, while $I$ and $A$ are increasing functions of $F$. Hence we have

$$
A_{1} \leq A_{2}, I_{1} \leq I_{2} \text { and } S_{1} \geq S_{2} .
$$

The two positive roots of (10) appear simultaneously as a double root $F_{1}=F_{2}$, which then splits into two simple roots. Hence, in the bifurcation state when $F_{1}=F_{2}$, the equilibrium $\mathcal{E} \mathcal{E}_{1}=\mathcal{E} \mathcal{E}_{2}$ appears and then splits into the two distinct equilibria $\mathcal{E E}_{1}$ and $\mathcal{E E}_{2}$. Since the constant term of (10) is strictly positive, this bifurcation is bounded away from the PFE. The PFE does not undergo any bifurcation and, as mentioned above, it is always asymptotically stable.

We perform numerical simulations using nonstandard finite difference schemes [2] to solve systems (1) and (33).

In all numerical simulations of model (1) we observe two qualitatively different cases and the transition (bifurcation) from one to the other. The 
one case is when PFE is the only equilibrium of the system. In this case we observe that all solution converge to PFE, that is PFE is globally asymptotically stable on $\mathbb{R}_{+}^{4}$. An illustrative example is given Figure 2 with value of the parameters given in Table $\amalg$.

TABLE I

Parameter Values USEd in Figure 2

\begin{tabular}{|c|c||c|c|}
\hline Parameter & Value & Parameter & Value \\
\hline$\Lambda$ & 1.1000 & $\lambda$ & 0.5000 \\
$\gamma$ & 0.5000 & $\beta$ & 1.0000 \\
$\sigma$ & 0.4000 & $\rho$ & 0.2000 \\
$\delta$ & 0.1000 & $M$ & 10.000 \\
$\alpha$ & 0.5100 & $d$ & 0.5000 \\
\hline
\end{tabular}

The second case is when the model has two positive equilibria. In the simulations presented in Figure 3, $\mathcal{E E}_{2}$ is stable and attracting, while $\mathcal{E E}_{1}$ is unstable (saddle point). Table II contains the parameter values used for the simulations in Figure 3 . The solutions that are initiated below $\mathcal{E}_{1}$ in the $(A, F, I)$-space converge to the PFE. Solutions $\phi_{1}$ and $\phi_{2}$ are initiated at $\mathcal{E} \mathcal{E}_{1}$ with altered value of $S$, $S_{0}=2$ and $S_{0}=20$ respectively. These values are below and above the PFE value of $S$, respectively. We observe that $\phi_{1}$ converges to the PFE, while $\phi_{2}$ increases and eventually converges to $\mathcal{E} \mathcal{E}_{2}$. The unstable equilibrium is typically very close to the PFE, so that the basin of attraction of the PFE is relatively small. Nevertheless, it contains the whole nonnegative $S$-axis.

TABLE II

PARAMETER VAlues USED IN Figure 3 .

\begin{tabular}{|c|c||c|c|}
\hline Parameter & Value & Parameter & Value \\
\hline$\Lambda$ & 1.0000 & $\lambda$ & 0.5000 \\
$\gamma$ & 0.9000 & $\beta$ & 5.0000 \\
$\sigma$ & 0.4000 & $\rho$ & 0.1000 \\
$\delta$ & 0.1000 & $M$ & 100.000 \\
$\alpha$ & 0.0450 & $d$ & 0.1000 \\
\hline
\end{tabular}

Due to the complexity of the model, we could not obtain theoretically a general result for the observed properties of the positive equilibria, or alternatively the global asymptotic stability of the PFE. However, we derive in the next two sections sufficient conditions for the two practically important properties: extinction and persistence of the pathogen.

\section{SUFFICIENT CONDITIONS FOR GLOBAL ASYMPTOTIC STABILITy OF PFE}

We prove sufficient conditions for global asymptotic stability of PFE using LaSalle's Invariance Principle [11, Theorem 2].

Theorem 1. The PFE of model (1) is globally asymptotically stable on $\mathbb{R}_{+}^{4}$ if either condition a) or condition b) below hold:

$$
\begin{aligned}
& \text { a) } \frac{\lambda \beta \gamma^{2} \Lambda^{2}}{16 d^{2}(\alpha+d) \delta M} \leq 1 \\
& \text { b) } \beta<\alpha+d \text { and } \\
& \frac{\lambda \beta^{2} \gamma^{2} \Lambda^{2}}{4 d^{2} \delta M(\beta+\alpha+d)^{2}} \leq 1 .
\end{aligned}
$$

Proof: Taking into account the inequalities (4), it is sufficient to consider the system (1) on the domain

$$
\Omega=\left\{x \in \mathbb{R}_{+}^{4}: S+I \leq \frac{\Lambda}{d}\right\} .
$$

We consider on $\Omega$ the function

$$
V(x)=A+F+\frac{\xi}{2} I^{2},
$$

where $\xi$ is a positive constant with value yet to be determined. We have

$$
\begin{aligned}
\dot{V}(x)= & \dot{A}+\dot{F}+\xi I \dot{I} \\
= & \lambda A(\gamma I-A)-\xi(\alpha+d) I^{2} \\
& +\xi \frac{\beta F}{M+F} S I-\delta F .
\end{aligned}
$$

Since the first term is a quadratic of $A$ it obtains it largest value when $A=\frac{1}{2} \gamma I$. Using also that $F \geq 0$ and

$$
S I \leq\left(\frac{S+I}{2}\right)^{2} \leq \frac{\Lambda^{2}}{4 d^{2}}
$$

we have

$$
\dot{V}(x) \leq\left(\frac{\lambda \gamma^{2}}{4}-\xi(\alpha+d)\right) I^{2}+\left(\frac{\xi \beta \Lambda^{2}}{4 d^{2} M}-\delta\right) F
$$




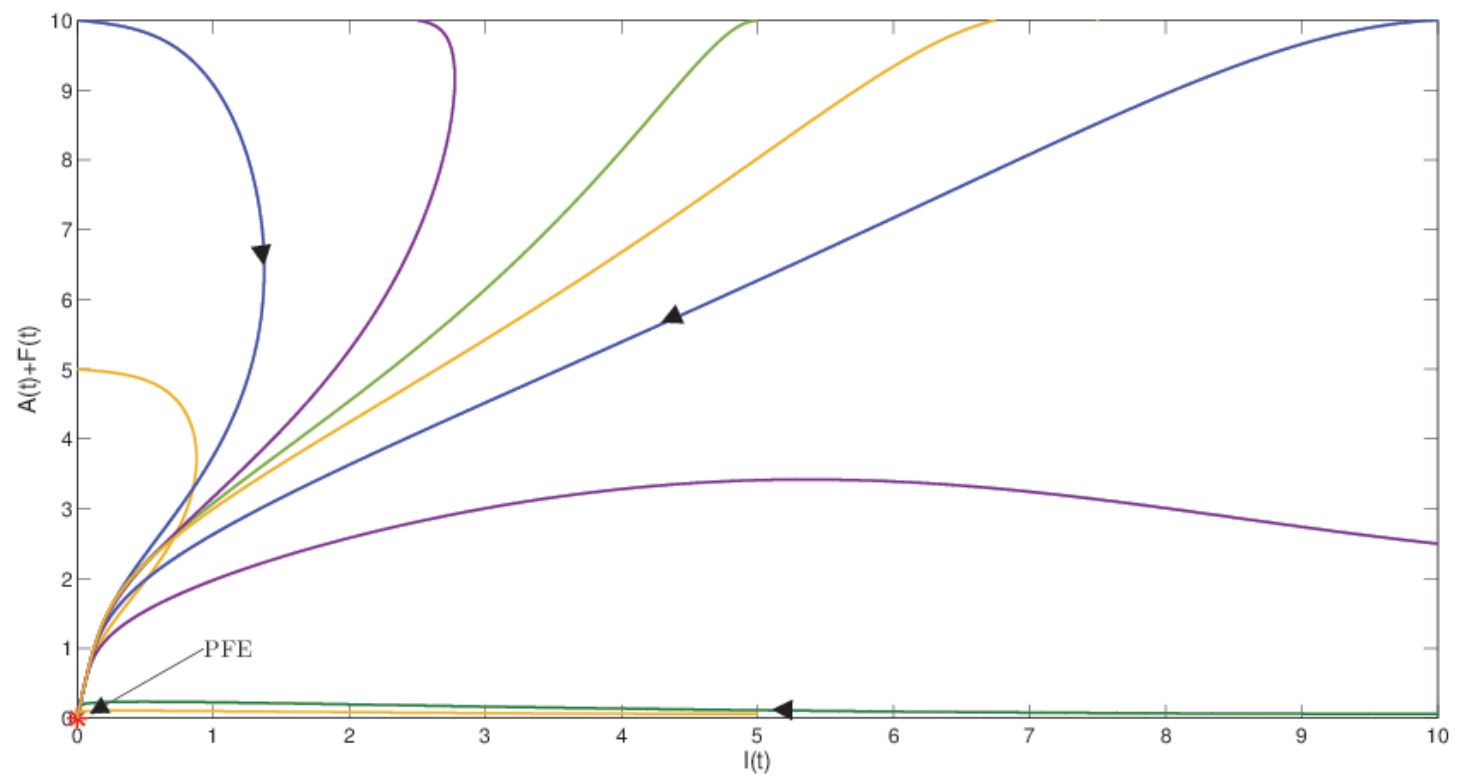

Fig. 2. Illustration of the case of global asymptotic stability of the PFE.

The coefficient of $I^{2}$ is nonpositive if and only if

$$
\xi \geq \frac{\lambda \gamma^{2}}{4(\alpha+d)}
$$

Similarly, the coefficient of $F$ is nonpositive if and only if

$$
\xi \leq \frac{4 d^{2} M \delta}{\beta \Lambda^{2}}
$$

A constant $\xi$ satisfying (15) and $(16)$ exists if and only if

$$
\frac{\lambda \gamma^{2}}{4(\alpha+d)} \leq \frac{4 d^{2} M \delta}{\beta \Lambda^{2}},
$$

or, equivalently,

$$
\frac{\lambda \beta \gamma^{2} \Lambda^{2}}{16 d^{2}(\alpha+d) \delta M} \leq 1,
$$

that is if and only if condition a) holds. Hence, if a) holds, we can select $\xi$ such that $\dot{V}(x) \leq 0$ for $x \in \Omega$. Let $E=\{x \in \Omega: \dot{V}(x)=0\}$. According to LaSalle Invariance Principle, all solutions converge to the largest invariant set $\mathcal{M}$ of (1) which is contained in $E$.

If the inequality in (17) is strict, then $\xi$ can be selected in such a way that the coefficients in (14) are negative. Hence, $\dot{V}(x)=0$ only if $I=F=0$ and then $A=0$ as well. Hence,

$$
E=\left\{(0,0, S, 0): 0 \leq S \leq \frac{\Lambda}{d}\right\}
$$

The largest invariant set in $E$ given in $(18)$ is $\mathcal{M}=$ $\{$ PFE $\}$.

If the inequality in (17) holds as equality, then the right hand side of (14) is zero irrespective of the values of $I$ and $F$. However, if $I$ or $F$ is not zero, then the respective inequalities leading to (14) should be satisfied as equalities. This process leads enlargement of $E$ in $(18)$ by an isolated point $\left(\frac{\gamma \Lambda}{4 d}, 0, \frac{\Lambda}{2 d}, \frac{\Lambda}{2 d}\right)$ and we again have $\mathcal{M}=\{\mathbf{P F E}\}$.

Therefore, by the LaSalle Invariance Principle all solutions converge to PFE.

b) The condition $\beta<\alpha+d$ provides for slightly sharper upper bound of $S I$ and hence slightly weaker condition on the parameters. 


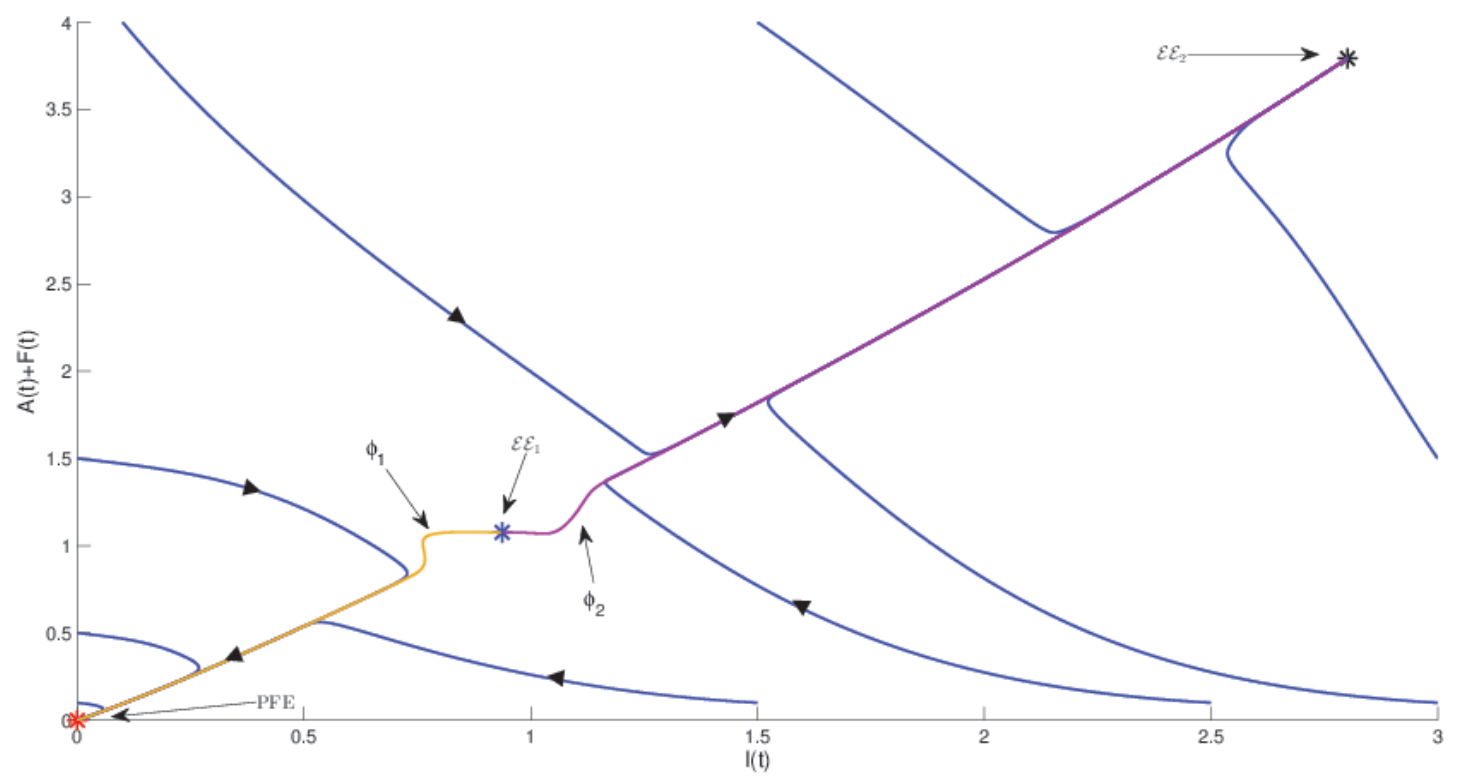

Fig. 3. If solutions of model (1) are initiated 'close' to the PFE convergence to this equilibrium occurs. Solutions that are initiated from the 'smaller' endemic equilibrium converge to either the PFE or the 'larger' equilibrium, dependent on the initial density of the susceptible compartment.

From the last equation of the model (1) we have Hence,

$$
\begin{aligned}
\frac{d I}{d t} & \leq \beta S-(\alpha+d) I \\
& \leq \beta(S+I)-(\beta+\alpha+d) I \\
& \leq \frac{\beta \Lambda}{d}-(\beta+\alpha+d) I
\end{aligned}
$$

Therefore

$$
\limsup _{t \rightarrow+\infty} I(t) \leq \frac{\beta \Lambda}{d(\beta+\alpha+d)} .
$$

$$
\begin{aligned}
S I & \leq\left(\frac{\Lambda}{d}-I\right) I \\
& \leq\left(\frac{\Lambda}{d}-\frac{\beta \Lambda}{d(\beta+\alpha+d)}\right) \frac{\beta \Lambda}{d(\beta+\alpha+d)} \\
& =\frac{\beta(\alpha+d) \Lambda^{2}}{(\beta+\alpha+d)^{2} d^{2}} .
\end{aligned}
$$

Then, following the same method as for a), in place of (17) we obtain the second inequality in b).

The parameter values given in Table I] satisfy

Hence, in the investigation of the asymptotic behavior of the system we can consider only the subset of $\Omega$ where

$$
I \leq \frac{\beta \Lambda}{d(\beta+\alpha+d)} .
$$

The inequality $\beta<\alpha+d$ implies that

$$
\frac{\beta \Lambda}{d(\beta+\alpha+d)} \leq \frac{\Lambda}{2 d} .
$$
both condition (a) and condition (b) of Theorem 1 . Hence, the global asymptotic stability of PFE observed earlier in Figure 2 can be deduced from either (a) or (b). Another illustration is given on Figure 4. The model parameters, given in Table III. satisfy condition (a), but not condition (b) in Theorem 1. This is sufficient to deduce the global asymptotic stability of PFE seen in Figure 4

We need to remark the conditions (a) and (b) are each only sufficient, but not necessary for the 
R. Anguelov, R. Bekker, Y. Dumont, Bi-stable dynamics of a host-pathogen model

TABLE III

PARAMETER VALUES USED IN Figure 4

\begin{tabular}{|c|c||c|c|}
\hline Parameter & Value & Parameter & Value \\
\hline$\Lambda$ & 0.9000 & $\lambda$ & 0.5000 \\
$\gamma$ & 0.5000 & $\beta$ & 1.0000 \\
$\sigma$ & 0.2000 & $\rho$ & 0.3000 \\
$\delta$ & 0.2900 & $M$ & 5.000 \\
$\alpha$ & 0.0600 & $d$ & 0.1500 \\
\hline
\end{tabular}

global stability of the PFE. The parameter values given in Table IV] satisfy neither of the conditions in Theorem 1. Yet, global asymptotic stability of PFE can be observed in the simulations presented in Figure 5 ,

TABLE IV

PARAMETER VALUES USED IN Figure 5

\begin{tabular}{|c|c||c|c|}
\hline Parameter & Value & Parameter & Value \\
\hline$\Lambda$ & 1.1000 & $\lambda$ & 0.6900 \\
$\gamma$ & 0.6000 & $\beta$ & 1.5000 \\
$\sigma$ & 0.4000 & $\rho$ & 0.5000 \\
$\delta$ & 0.2000 & $M$ & 5.000 \\
$\alpha$ & 0.0100 & $d$ & 0.3000 \\
\hline
\end{tabular}

\section{SUFFICIENT CONDITIONS FOR PERSISTENCE}

We derive sufficient conditions for persistence using the theory of monotone systems. The system (1) is not monotone. We construct an auxiliary system about the vector $y=(A, F, I)^{T}$ which is monotone. From the third equation of (1) we have

$$
\Lambda-(d+\beta) S \leq \frac{d S}{d t} \leq \Lambda-d S
$$

Then, it follows that for every solution of (1) it holds

$$
\frac{\Lambda}{\beta+d} \leq \liminf _{t \rightarrow+\infty} S(t) \leq \limsup _{t \rightarrow+\infty} S(t) \leq \frac{\Lambda}{d} .
$$

Hence, for the asymptotic properties of the solutions of (1) it is sufficient to consider the subset of $\Omega$ where

$$
\frac{\Lambda}{\beta+d} \leq S \leq \frac{\Lambda}{d}
$$

In this subdomain we consider the following system for $y=(A, F, I)^{T}$ :

$$
\frac{d y}{d t}=h(y):=\left(\begin{array}{c}
\lambda A(\gamma I-A)-\sigma A \\
-\delta F+\sigma A-\frac{\rho \Lambda}{d} F \\
\frac{\beta F}{M+F} \frac{\Lambda}{\beta+d}-(\alpha+d) I
\end{array}\right) .
$$

Let us recall that function $h$ is said to satisfy the Kamke condition if $h_{i}$ is increasing in $y_{j}$ for $i \neq j$. The Kamke condition implies that the respective system of ODEs is monotone with respect to the initial condition or shortly monotone, [16, Section 3.1]. The Jacobian of $h$ is

$$
J_{h}=\left(\begin{array}{ccc}
\lambda \gamma I-2 \lambda A-\sigma & 0 & \lambda \gamma A \\
\sigma & -\delta-\frac{\rho \Lambda}{d} & 0 \\
0 & \frac{\beta M \Lambda}{(\beta+d)(M+F)^{2}} & -(\alpha+d)
\end{array}\right) .
$$

Since the nondiagonal entries of $J_{h}$ are nonnegative, the system (21) is monotone. Moreover, since the system is irreducible in the interior of $\mathbb{R}_{+}^{3}$, it is strongly monotone, [16, Theorem 4.1.1]. Let us recall that a system of the form (21) is called strongly monotone if for any two solution $y^{(1)}$ and $y^{(2)}$

$$
\begin{aligned}
y^{(1)}(0)<y^{(2)}(0) \Longrightarrow & y_{i}^{(1)}(t)<y_{i}^{(2)}(t), t>0, \\
& i=1,2,3 .
\end{aligned}
$$

Our interest in the system (21) is motivated by the fact that its solutions provide lower bounds for the coordinates $A, F$, and $I$ of the solutions of (1). This will be shown later by using differential inequalities given in [18] for systems of ODEs with quasi-monotone right hand side. Hence, we carry out first the asymptotic analysis of (21).

To find the equilibria of (21), we set the right hand side to zero:

$$
\begin{aligned}
& \lambda A(\gamma I-A)-\sigma A=0 \\
& -\delta F+\sigma A-\frac{\rho \Lambda}{d} F=0 \\
& \frac{\beta F}{M+F} \frac{\Lambda}{\beta+d}-(\alpha+d) I=0
\end{aligned}
$$

The origin, $\mathbf{0}$ is an equilibrium. To find the nonzero equilibria, we multiply the first equation by $\frac{\sigma}{\lambda}$ and add it to the second one to eliminate $A$. We 


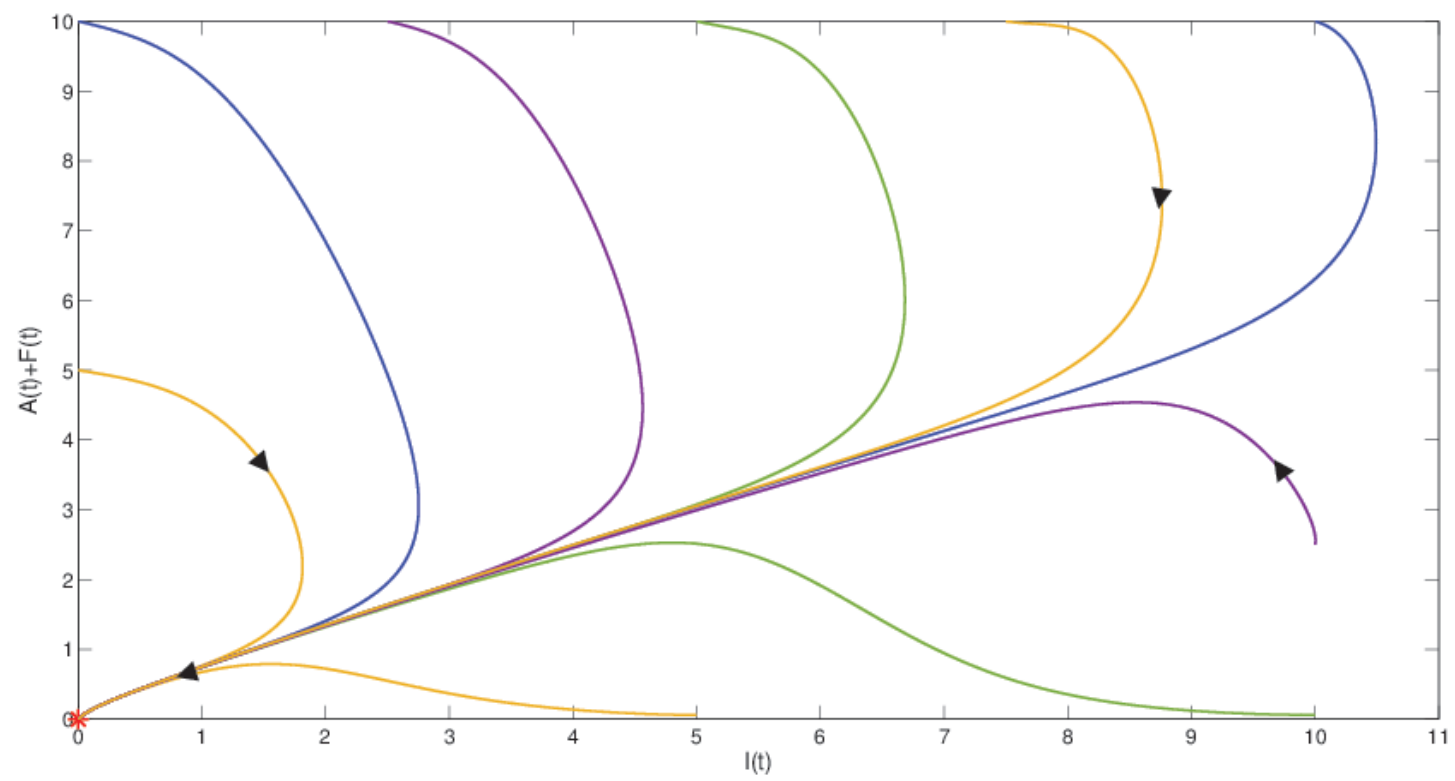

Fig. 4. Illustration of the global stability of the PFE of the host-pathogen model when only condition (a) in Theorem 1 holds.

multiply the third equation by $\frac{\gamma \sigma}{\alpha+d}$ and add it to the second one to eliminate $I$. Hence, we obtain

$$
\begin{aligned}
& \varphi(y):=\frac{\sigma}{\lambda} h_{1}(y)+h_{2}+\frac{\gamma \sigma}{\alpha+d} h_{3}(y)= \\
& -\left(\delta+\frac{\rho \Lambda}{d}\right) F-\frac{\sigma^{2}}{\lambda}+\frac{\gamma \sigma \beta \Lambda F}{(\alpha+d)(\beta+d)(M+F)}=0
\end{aligned}
$$

Equation 26 is equivalent to the quadratic equation

$$
\begin{aligned}
& \left(\delta+\frac{\rho \Lambda}{d}\right) F^{2}+ \\
& \left(M\left(\delta+\frac{\rho \Lambda}{d}\right)+\frac{\sigma^{2}}{\lambda}-\frac{\gamma \sigma \beta \Lambda}{(\beta+d)(\alpha+d)}\right) F \\
& +\frac{M \sigma^{2}}{\lambda}=0
\end{aligned}
$$

The equation (27) has two positive real roots if and only if the coefficient of $F$ is negative and the discriminant is positive, that is

$$
\begin{gathered}
M\left(\delta+\frac{\rho \Lambda}{d}\right)+\frac{\sigma^{2}}{\lambda}-\frac{\gamma \sigma \beta \Lambda}{(\beta+d)(\alpha+d)}<0 \\
\Delta=\left(M\left(\delta+\frac{\rho \Lambda}{d}\right)+\frac{\sigma^{2}}{\lambda}-\frac{\gamma \sigma \beta \Lambda}{(\beta+d)(\alpha+d)}\right)^{2} \\
\quad-\frac{4 M \sigma^{2}}{\lambda}\left(\delta+\frac{\rho \Lambda}{d}\right)>0
\end{gathered}
$$

Assuming that conditions (28)-29) hold, we denote by $\tilde{F}_{1}$ and $\tilde{F}_{2}, \tilde{F}_{1}<\tilde{F}_{2}$, the roots of 27 ) and by $\tilde{\mathcal{E}}_{1}=\left(\tilde{A}_{1}, \tilde{F}_{1}, \tilde{I}_{1}\right)^{T}$ and $\tilde{\mathcal{E}}_{2}=\left(\tilde{A}_{2}, \tilde{F}_{2}, \tilde{I}_{2}\right)^{T}$ the corresponding equilibria of (21). where

$$
\begin{aligned}
A_{i} & =\frac{1}{\sigma}\left(\delta+\frac{\rho \Lambda}{d}\right) F_{i}, i=1,2, \\
I_{i} & =\frac{1}{\gamma}\left(A_{i}+\sigma\right), i=1,2 .
\end{aligned}
$$

Considering the expressions 30 and 31, we have $\mathbf{0}<<\tilde{\mathcal{E}}_{1}<<\tilde{\mathcal{E}}_{2}$.

Theorem 2. Let conditions (28)-(29) hold. Then for every solution $y(t)$ of (21) we have

$$
y(0)>\tilde{\mathcal{E}}_{1} \Longrightarrow \liminf _{t \rightarrow+\infty} y(t) \geq \tilde{\mathcal{E}}_{2} \text {. }
$$




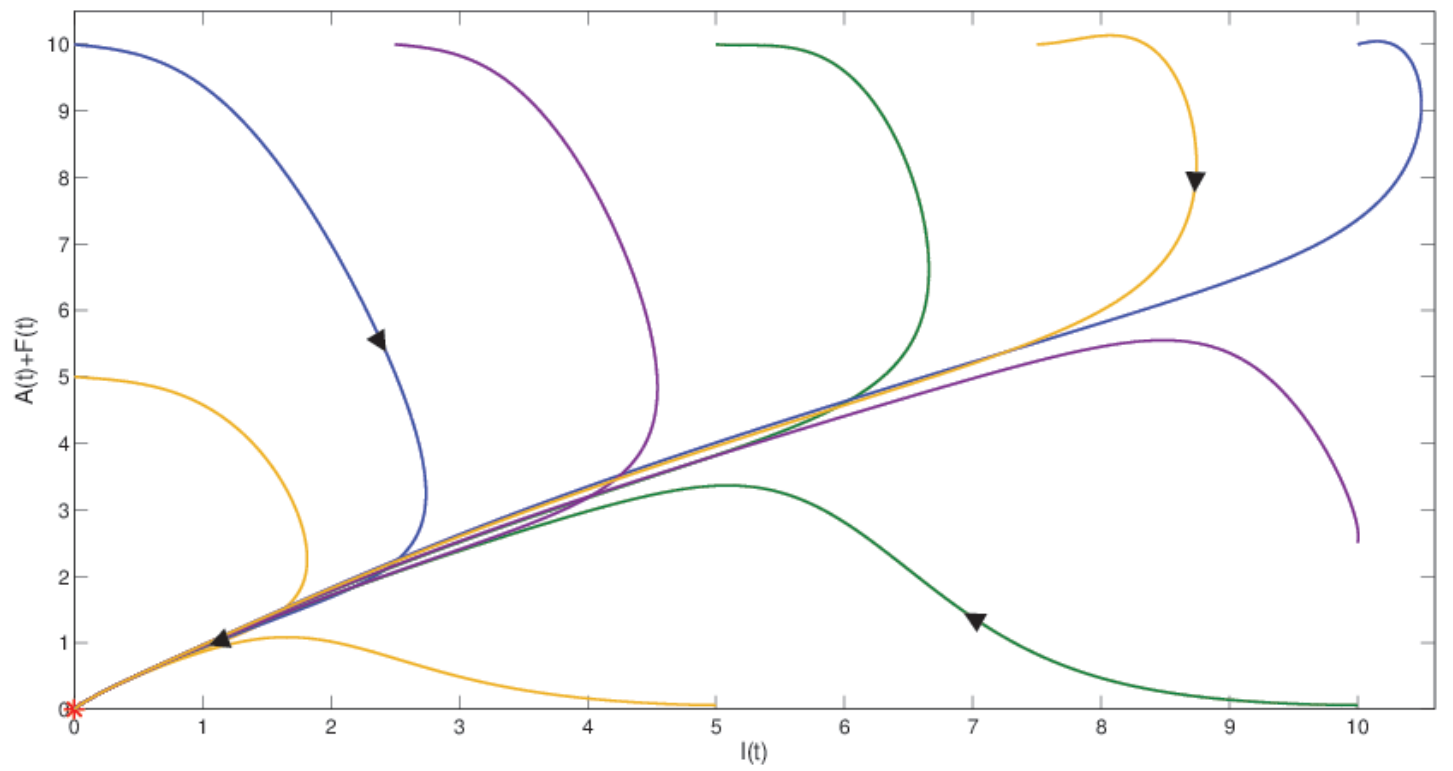

Fig. 5. Illustration that PFE of the host-pathogen model may be globally asymptotically stable when neither of the condition in Theorem 1 holds.

Proof: It is easy to see that the equilibrium $\mathbf{0}$ is asymptotically stable, indeed the eigenvalues of $J_{h}(\mathbf{0}), \xi_{1}=-\sigma, \xi_{2}=-\left(\delta+\frac{\rho \Lambda}{d}\right)$ and $\xi_{3}=$ $-(\alpha+d)$ are all negative.

We consider the order interval $\left[\mathbf{0}, \tilde{\mathcal{E}}_{1}\right]$. It follows from [16, Theorem 2.2.2], that the solutions initiated in this interval, excluding the end points, either all converge to $\mathbf{0}$ or all converge to $\tilde{\mathcal{E}}_{1}$. Since $\mathbf{0}$ is asymptotically stable, this implies that all solutions converge to $\mathbf{0}$. The Jacobian of $h$ at $\tilde{\mathcal{E}}_{1}$ after some simplifications is:

$J_{h}\left(\tilde{\mathcal{E}}_{1}\right)=\left(\begin{array}{ccc}-\lambda A^{*} & 0 & \lambda \gamma A^{*} \\ \sigma & -\delta-\frac{\rho \Lambda}{d} & 0 \\ 0 & \frac{\beta M \Lambda}{(\beta+d)\left(M+\tilde{F}_{1}\right)^{2}} & -(\alpha+d)\end{array}\right)$,

Since the nondiagonal entries of $J_{h}\left(\tilde{\mathcal{E}}_{1}\right)$ are nonnegative and the matrix is irreducible, it follows from the theory of nonnegative matrices [4, Theorem 2.1.4] that $J_{h}\left(\tilde{\mathcal{E}}_{1}\right)$ has eigenvector $v$ with positive coordinates and associated eigenvalue $\xi$, which is a real number. Since $\tilde{\mathcal{E}}_{1}$ is repelling in $\left[\mathbf{0}, \tilde{\mathcal{E}}_{1}\right]$, we have that $\xi \geq 0$. We will show that $\xi>0$. Assume the opposite, namely $\xi=0$. Then it is easy to compute that $w=\left(\frac{\sigma}{\gamma \tilde{A}_{1}}, 1, \frac{\sigma \gamma}{\alpha+d}\right)^{T}$ is a left eigenvector. Then, using the expression for $\varphi$ in 25 and that $h\left(\tilde{\mathcal{E}}_{1}\right)=0$ we have

$$
\nabla \varphi\left(\tilde{\mathcal{E}}_{1}\right)=w^{T} J_{h}\left(\tilde{\mathcal{E}}_{1}\right)=\mathbf{0} .
$$

The first and the third coordinates of $\nabla \varphi(y)$ are always zero since $\varphi$ does not depend on $A$ and $I$. The fact that $\frac{\partial \varphi\left(\tilde{\mathcal{E}}_{1}\right)}{\partial F}=0$ implies that the equation (26), or, equivalently 27, has a double root. This contradicts (29). Therefore $\xi>0$.

Next, we consider the order interval $\left[\tilde{\mathcal{E}}_{1}, \tilde{\mathcal{E}}_{2}\right]$. Again following [16, Theorem 2.2.2], that the solutions initiated in this interval, excluding the end points, either all converge to $\tilde{\mathcal{E}}_{1}$ or all converge to $\tilde{\mathcal{E}}_{2}$. Considering that $\tilde{\mathcal{E}}_{1}$ is repelling in the direction of the positive vector $v$, we conclude that all solutions converge to $\tilde{\mathcal{E}}_{2}$.

Let $y(t)$ be any solution of (21) such that $y(0)>\tilde{\mathcal{E}}_{1}$. Consider the solution of 21 with initial condition $z(0)=\min \left\{y(0), \tilde{\mathcal{E}}_{2}\right\}$. Since $z(0) \in\left[\tilde{\mathcal{E}}_{1}, \tilde{\mathcal{E}}_{2}\right]$ and $z(0)>\tilde{\mathcal{E}}_{1}$, we have

$$
\lim _{t \rightarrow+\infty} z(t)=\tilde{\mathcal{E}}_{2} \text {. }
$$


Then using that $y(0) \geq z(0)$ and the monotonicity of the system 21 we have $y(t) \geq z(t), t \geq 0$. Therefore

$$
\liminf _{t \rightarrow+\infty} y(t) \geq \lim _{t \rightarrow+\infty} z(t)=\tilde{\mathcal{E}}_{2}
$$

Using Theorem 2 for the auxiliary system (21) we prove the following theorem for the original model (1).

Theorem 3. Let conditions (28) and (29) hold. Then, for any solution of (1) we have that if

$$
A(0)>\tilde{A}_{1}, F(0)>\tilde{F}_{1}, I(0)>\tilde{I}_{1},
$$

then

$$
\begin{aligned}
& \liminf _{t \rightarrow+\infty} A(t) \geq \tilde{A}_{2}, \\
& \liminf _{t \rightarrow+\infty} F(t) \geq \tilde{F}_{2}, \\
& \liminf _{t \rightarrow+\infty} I(t) \geq \tilde{I}_{2} .
\end{aligned}
$$

Proof: As discussed earlier, it is sufficient to consider the subdomain, where 20 holds. Let $x(t)$ be a solution of (2) such that $A(0), F(0)$, $I(0)$ satisfy 32 and $S(0) \in\left[\frac{\Lambda}{\beta+d}, \frac{\Lambda}{d}\right]$. From 19 it follows that $S(t) \in\left[\frac{\Lambda}{\beta+d}, \frac{\Lambda}{d}\right]$ for $t \geq 0$. Then the coordinates $A(t), F(t)$, and $I(t)$ satisfy

$$
\frac{d}{d t}\left(\begin{array}{l}
A(t) \\
F(t) \\
I(t)
\end{array}\right)=\left(\begin{array}{l}
f_{1}(x) \\
f_{2}(x) \\
f_{4}(x)
\end{array}\right) \geq h\left(\begin{array}{c}
A(t) \\
F(t) \\
I(t)
\end{array}\right)
$$

Using that $h$ is quasi-monotone and applying [18, Chapter 2, Section 12.X], we obtain that the vector function $(A(t), F(t), I(t))^{T}$ is bounded below by the solution of 21) with the same initial condition at $t=0$. Then, Theorem 2 implies that $\tilde{\mathcal{E}}_{2}$ is a lower bound for the limit inferior of $(A(t), F(t), I(t))^{T}$ as $t \rightarrow+\infty$, which proves the theorem.

Theorem 3 shows that if the initial invasion is sufficiently large the pathogen establishes itself at a level above $\tilde{\mathcal{E}}_{2}$. We should remark that it is not necessary to have initially all compartments $A$, $F$ and $I$ above $\tilde{\mathcal{E}}_{1}$. It is sufficient that at some future time they all exceed $\tilde{\mathcal{E}}_{1}$. For example, and as it can be also expected from biological point of view, the initial infection/infestation is brought in the compartment $F$. If this initial value of $F$ is sufficiently large that $A$ and $I$ increase above $\tilde{A}_{1}$ and $\tilde{I}_{1}$, while $F$ is still above $\tilde{F}_{1}$, the pathogen will persist eventually at least at a level of $\tilde{\mathcal{E}}_{2}$.

Theorem 3 is illustrated numerically by Figure 6. The initial conditions of the solutions in this figure were chosen specifically so that $\left(A_{0}, F_{0}, I_{0}\right) \geq \tilde{\mathcal{E}}_{1}$. Clearly any solution initiated at or above the level of $\tilde{\mathcal{E}}_{1} \approx(4.091,1.7241,0.2164)$ persists at a non-zero level above $\tilde{\mathcal{E}}_{2}$ for all time; and in fact converges to an equilibrium of model (1), at least for the parameter values in Table V] We verify that the data in Table V satisfies conditions (28)-(29) of Theorem 3. Indeed, the left hands sides of (28) and (29) are respectively negative and positive, so that inequalities in (28) and 29 hold.

Theorem 3 gives only sufficient conditions for persistence of pathogen. In Figure 7 , we show that the pathogen persist, in fact, the solutions converge to $\mathcal{E} \mathcal{E}_{2}$, even though the initial conditions do not satisfy the requirements of Theorem 3 .

TABLE V

PARAMETER VAlues USED IN Figures 6 and 7

\begin{tabular}{|c|c||c|c|}
\hline Parameter & Value & Parameter & Value \\
\hline$\Lambda$ & 0.9000 & $\lambda$ & 1.0000 \\
$\gamma$ & 23.52536 & $\beta$ & 5.0000 \\
$\sigma$ & 1.0000 & $\rho$ & 0.9000 \\
$\delta$ & 0.9000 & $M$ & 10.000 \\
$\alpha$ & 0.0010 & $d$ & 0.5500 \\
\hline
\end{tabular}

Our numerical investigations of model (1) have revealed that in addition to the PFE which is always locally attracting, there exists an equilibrium close to the PFE which is repelling, and an attracting equilibrium which is removed from the PFE. Although we have not proven this mathematically, the important properties of the model, namely extinction and persistence, have been proven under certain conditions.

\section{THE SPATIO-TEMPORAL HOST-PATHOGEN MODEL}

The model in Section [I] looks only at the temporal progression of an infection, and although this 


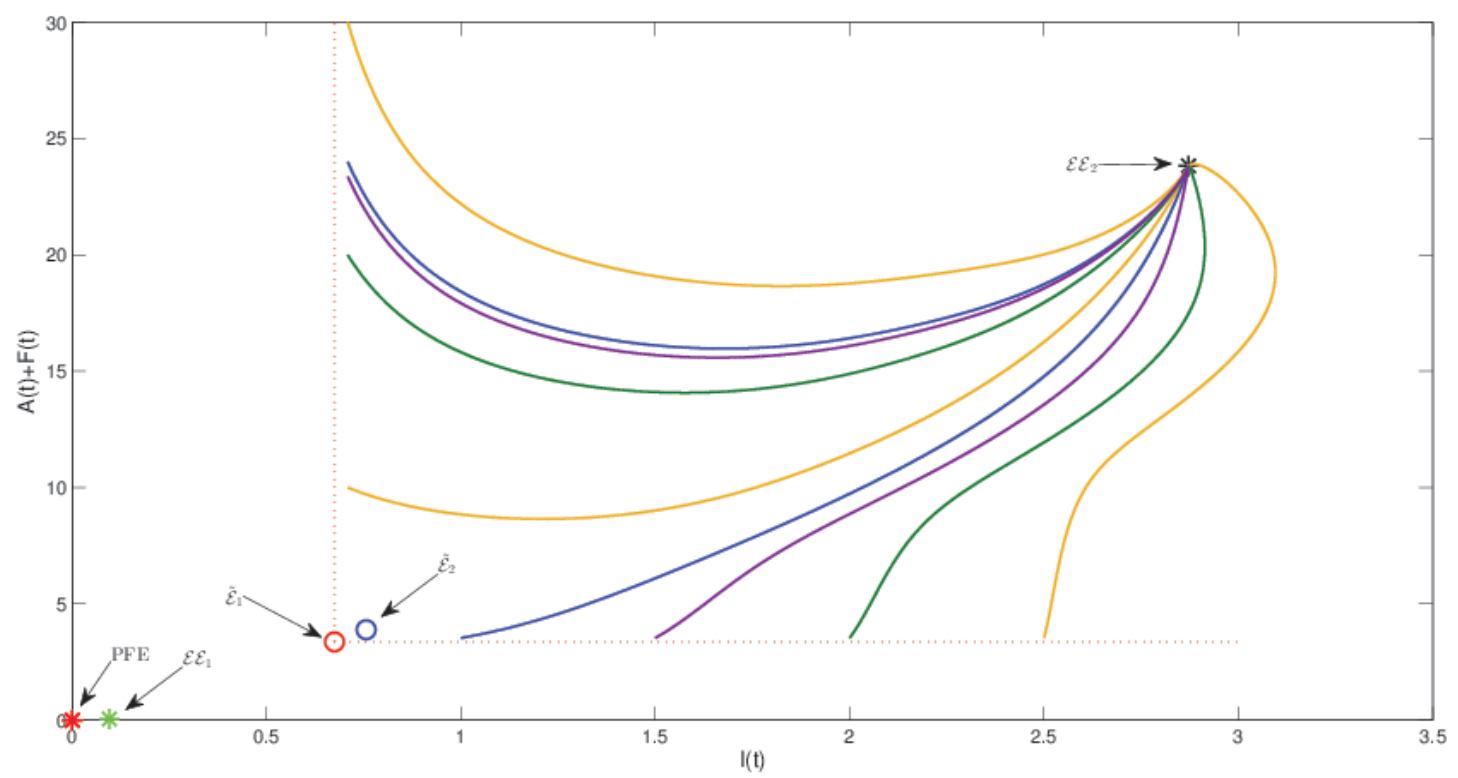

Fig. 6. Illustration of persistence of the infection as proven in Theorem 3 Observe that solutions of model (1) originating at the level of $\tilde{\mathcal{E}}_{1}$ remain non-zero for all time.

approach is acceptable under certain assumptions (such as a pathogen entering an entire field in a uniform manner), the model can be modified slightly to accommodate the spatial movement of pathogens through the field.

Diffusion has been used to model spatial spread in theoretical ecology since the latter half of the twentieth century [9], [15], with its use for modelling fungal growth being justified by the "observation that tip growth occurs to fill space and to capture nutrients" [5]. Davidson (1998) also noted that fungal growth "is, in the main, directed from areas of high hyphal density to areas of low hyphal density", and included diffusion in his model with the warning 'that this flux should not be viewed as the movement of existing biomass, but rather the propensity of new biomass to grow away from high density areas'. We reiterate this warning, and include diffusion to model the spatial growth of off-host pathogen in search of new hosts, with $\mu$ denoting the diffusion constant. Our Host-Pathogen spatio-temporal model is defined as follows:

$$
\left\{\begin{array}{l}
\frac{\partial A}{\partial t}=\lambda A(\gamma I-A)-\sigma A+\rho F S \\
\frac{\partial F}{\partial t}=-\delta F+\sigma A-\rho F S+\mu \Delta F \\
\frac{\partial S}{\partial t}=\Lambda-d S-\frac{\beta F}{M+F} S \\
\frac{\partial I}{\partial t}=\frac{\beta F}{M+F} S-(\alpha+d) I \\
\text { with } \\
A(x, 0) \geq 0, \quad F(x, 0) \geq 0 \\
S(x, 0) \geq 0, \quad I(x, 0) \geq 0 \\
\frac{\partial F}{\partial x}(-L, t)=0=\frac{\partial F}{\partial x}(L, t)
\end{array}\right.
$$

If the initial condition is spatially uniform, and taking the boundary conditions into account, then the solution is also spatially uniform, reducing it to a solution of the corresponding temporal system. The properties and long-term behaviour of the temporal model have been theoretically proven in Section II] and this chapter devotes itself to numerical investigation of the behaviour of 


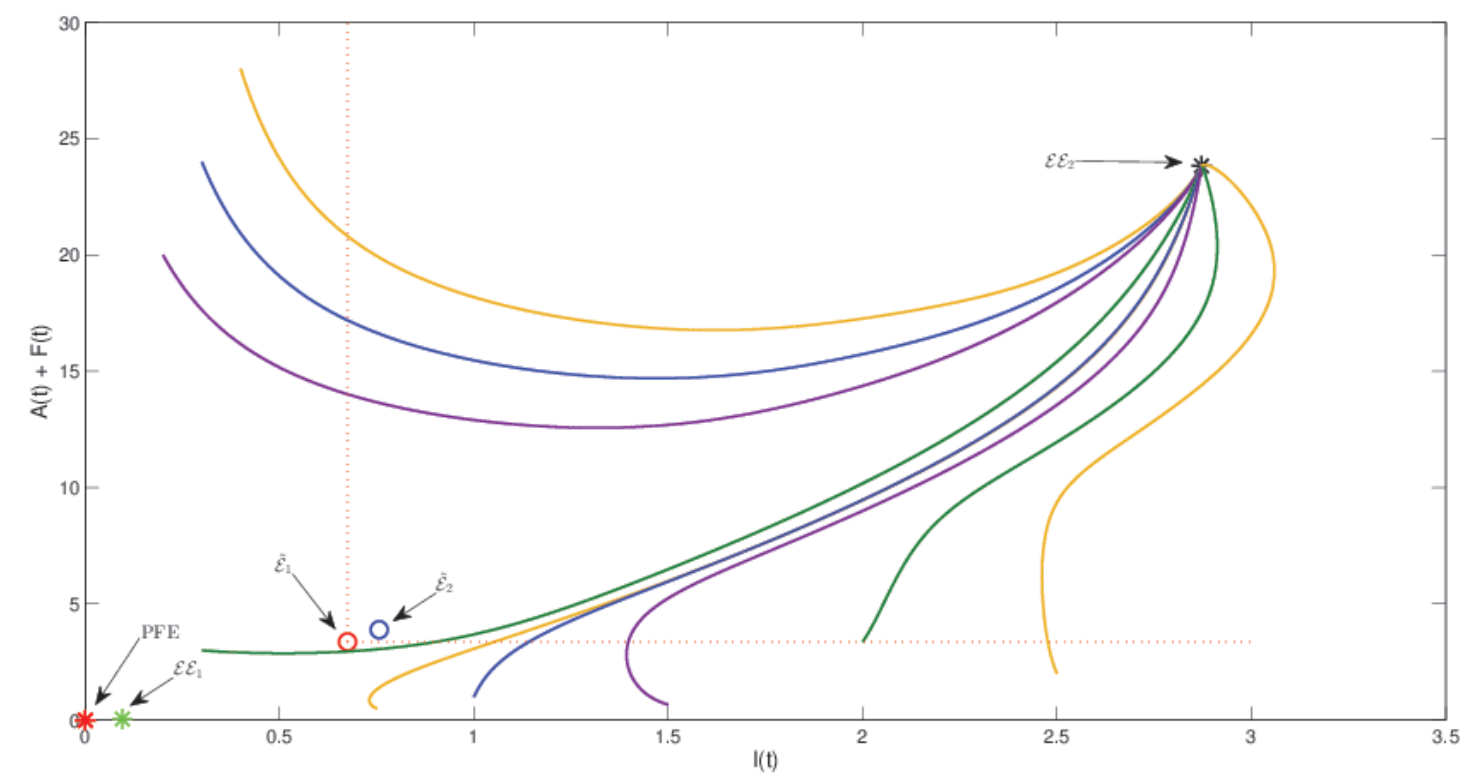

Fig. 7. Illustration of persistence of the infection when initial conditions do not satisfy conditions 32, in Theorem 3

solutions of system (33). Our interest is mainly in the practically relevant case where the pathogen is introduced in one location, and studying the dynamics of its propagation. In order to solve model (33) numerically, we use non-standard discretization coupled with a second order centralspace discretization [2].

\section{A. Numerical investigations}

1) Under the conditions for asymptotic stability obtained by application of LaSalle's Invariance Principle: The parameter values given in Table VI satisfy the conditions (12) which ensures the PFE of the temporal model is globally asymptotically stable. The details are given in Section IV We investigate whether the solutions of the spatiotemporal model behave in a similar fashion, using the diffusion constant $\mu=0.01$. Indeed, although convergence occurs over a long time period, the addition of diffusion does not result in observable change in the asymptotic properties of the steady state. In Figure 8, even assuming that the initial population has free pathogen over a quarter of the field, this does not result in the infection spreading.
TABLE VI

PARAMETER VALUES USED IN Figure 8

\begin{tabular}{|c|c||c|c|}
\hline Parameter & Value & Parameter & Value \\
\hline$\Lambda$ & 1.0000 & $\lambda$ & 0.4000 \\
$\gamma$ & 0.2000 & $\beta$ & 1.0000 \\
$\sigma$ & 0.0100 & $\rho$ & 0.4000 \\
$\delta$ & 0.1000 & $M$ & 100.000 \\
$\alpha$ & 0.0205 & $d$ & 0.1000 \\
\hline
\end{tabular}

TABLE VII

PARAmeter VAlues USED in Figure 9

\begin{tabular}{|c|c||c|c|}
\hline Parameter & Value & Parameter & Value \\
\hline$\Lambda$ & 1.0000 & $\lambda$ & 0.7000 \\
$\gamma$ & 0.4000 & $\beta$ & 1.0000 \\
$\sigma$ & 0.1000 & $\rho$ & 0.2000 \\
$\delta$ & 0.2000 & $M$ & 100.00 \\
$\alpha$ & 0.0100 & $d$ & 0.2000 \\
\hline
\end{tabular}

In Figure 9, we also provide an example where the conditions (12) are not satisfied and yet there is convergence to PFE (see also Table VII).

2) Parameter values for persistence of the infection: A monotone system, constructed to approximate the temporal host pathogen model from below was proven to admit two interior equilibria 

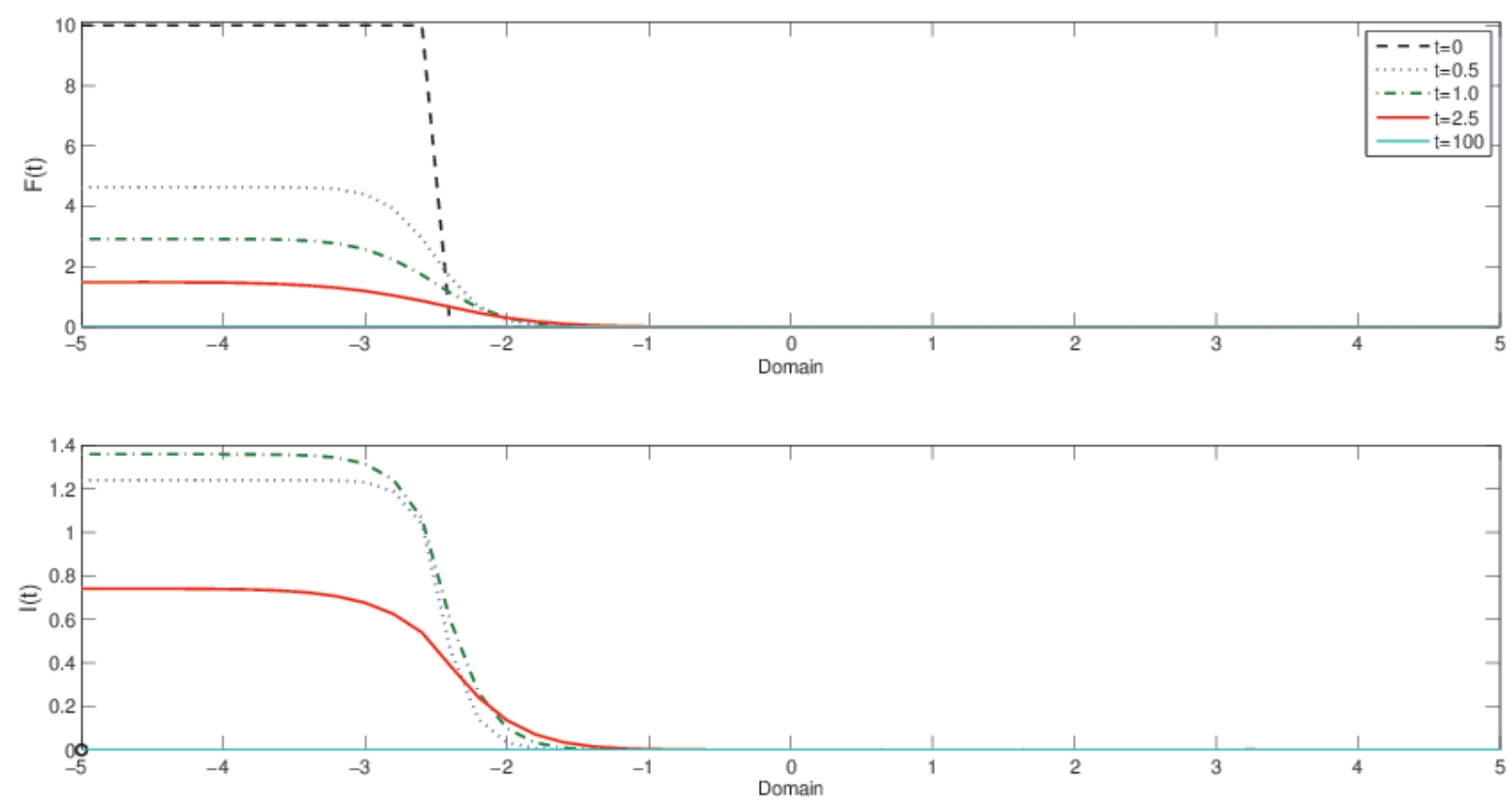

Fig. 8. The time evolution of pathogen and disease through the field at different times, using the parameter values that satisfy the conditions for stability of the PFE that were obtained by the application of LaSalle's Invariance Principle.
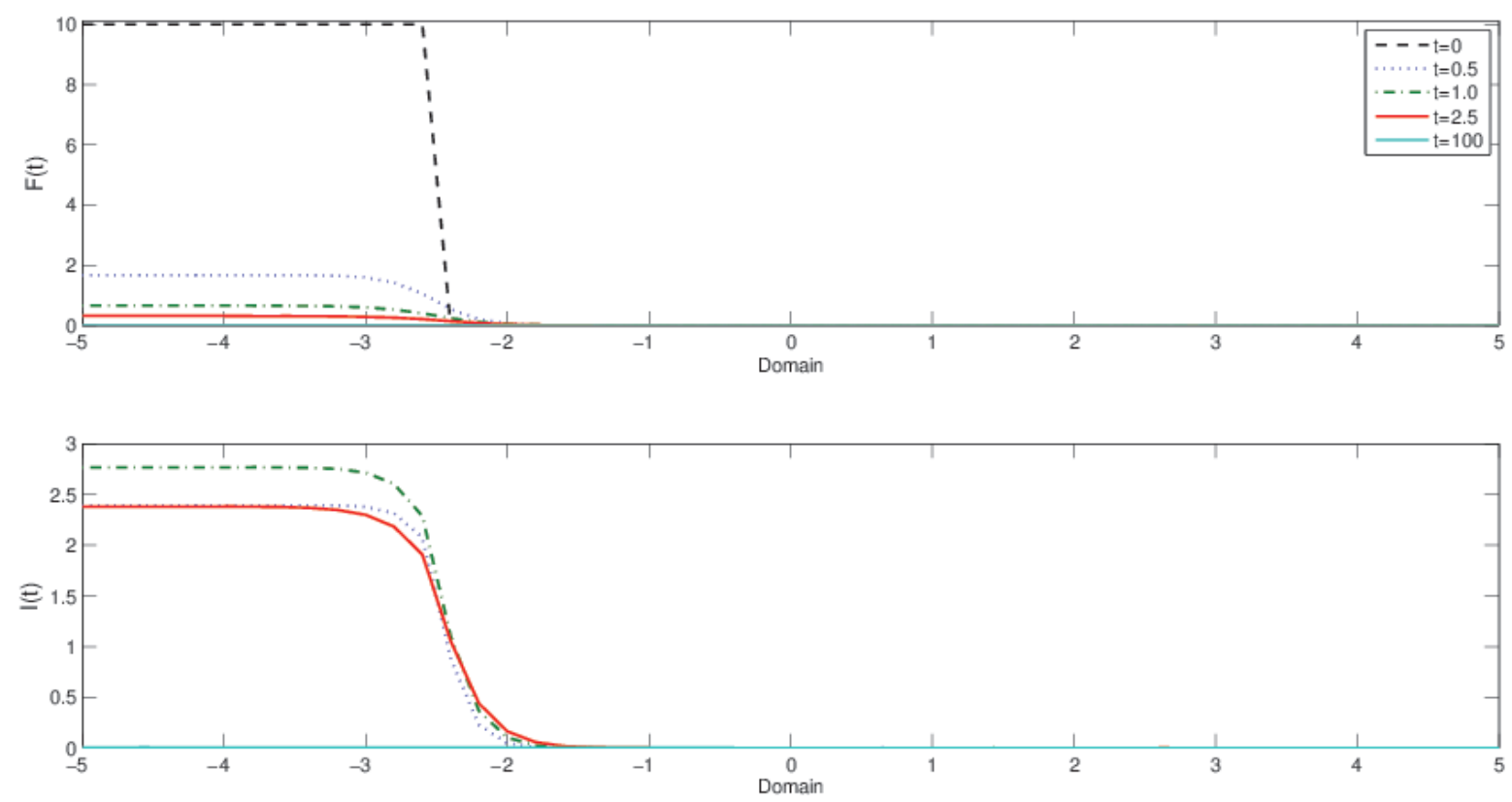

Fig. 9. The parameter values in Table VII do not satisfy condition for asymptotic stability of the PFE, however convergence to PFE is observed. 

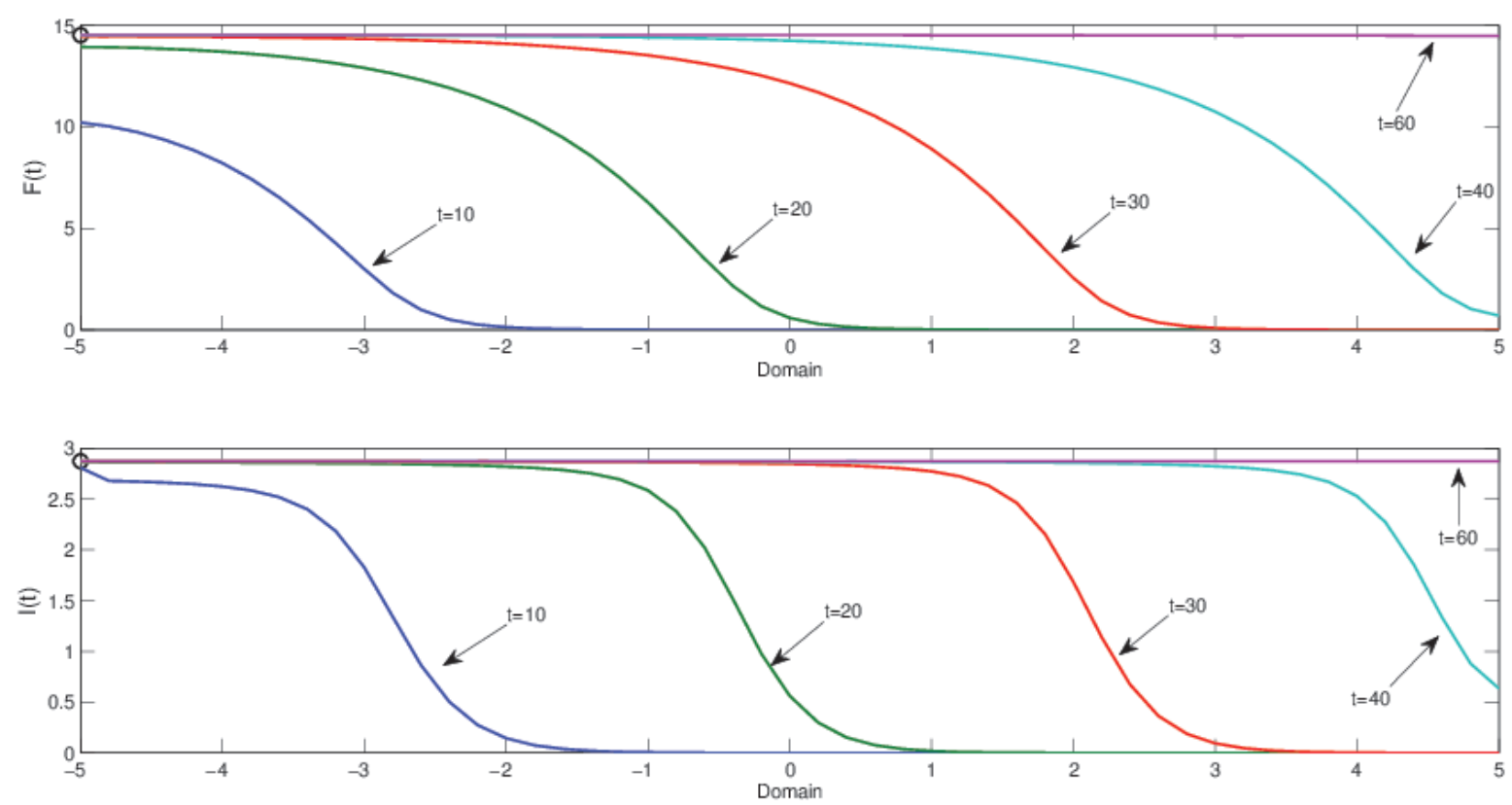

Fig. 10. When solutions of model $\sqrt{33}$ are initiated with pathogen and infectious hosts at the level of $\mathcal{E E}_{2}$, on the left boundary, a field of completely susceptible hosts will experience a travelling infection front. This front connects $\mathcal{E E}_{2}$ and the PFE.

in Section $\mathrm{V}$, denoted $\tilde{\mathcal{E}}_{1}$ and $\tilde{\mathcal{E}}_{1}$, with $\tilde{\mathcal{E}}_{1}<\tilde{\mathcal{E}}_{1}$. Additional conditions were derived, under which the pathogen persists. Indeed, it was found that solutions initiated at or above $\tilde{\mathcal{E}}_{1}$ and satisfying conditions (28) and (29), page 9, remain non-zero for all $t \geq 0$.

For the parameter values in Table VIII on page 15 , these conditions are satisfied. Indeed, we have

$$
\begin{aligned}
& \frac{M}{\gamma \sigma}\left(\delta+\frac{\rho \Lambda}{d}\right)+\frac{\sigma}{\gamma \lambda}-\frac{\beta \Lambda}{(\beta+d)(\alpha+d)} \\
& \quad \approx-0.4204<0, \\
& \Delta \approx 0.0053>0 .
\end{aligned}
$$

The equilibrium, $\tilde{\mathcal{E}}_{1}$ of the lower approximating system is:

$$
\tilde{\mathcal{E}}_{1} \approx(4.091,1.7241,0.2164) .
$$

The persistence of pathogen is illustrated in Figure 10. In fact, the solutions converge to $\mathcal{E E}_{2}$, although the stability properties of $\mathcal{E E}_{1}$ and $\mathcal{E E}_{2}$ have not been proven. How does the inclusion of diffusion affect this phenomenon? Solutions initiated at the level of $\mathcal{E} \mathcal{E}_{2}$ at the boundary exhibit a travelling infection front, the movement of which is driven by the increase in attached pathogen and infested hosts by the diffusion of the free pathogen (Figure 10). This behaviour suggests a possible control strategy: if the speed of the front can be sufficiently decreased, a percentage of the field would be saved from disease.

TABLE VIII

PARAMETER VALUeS USED IN Figure 10 .

\begin{tabular}{|c|c||c|c|}
\hline Parameter & Value & Parameter & Value \\
\hline$\Lambda$ & 0.9000 & $\lambda$ & 1.0000 \\
$\gamma$ & 23.52536 & $\beta$ & 5.0000 \\
$\sigma$ & 1.0000 & $\rho$ & 0.9000 \\
$\delta$ & 0.9000 & $M$ & 10.000 \\
$\alpha$ & 0.0010 & $d$ & 0.5500 \\
\hline
\end{tabular}

To this end, we investigate the relationship between $\mu$ and the wave speed $c$. The parameter values in Table VIII were again used, and a solution with $\left(A_{0}, F_{0}, I_{0}\right)$ taking the value of $\mathcal{E E}_{2}$ on the 


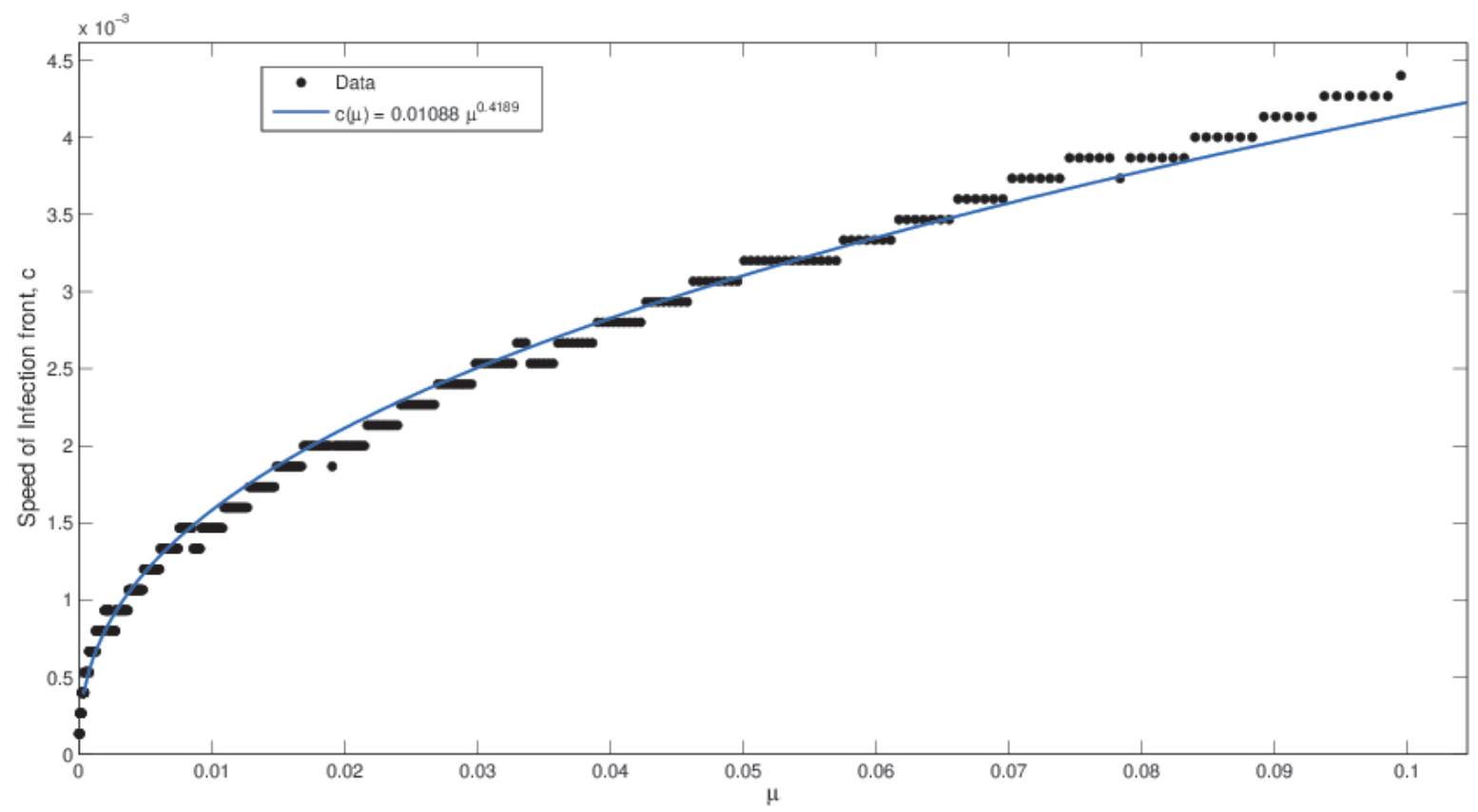

Fig. 11. The speed of the infection front for different values of $\mu$, for solutions of model (33) initiated with levels of inoculum and disease at the level of $\mathcal{E E}_{2}$ on the left boundary. The parameter values in Table VIII were used. Clearly the equation $c(\mu)=0.01088 \mu^{0.4189}$ fits the data well.

left boundary was considered. The diffusion constant $\mu$ was taken to be in the interval $\left[10^{-7}, 10^{-1}\right]$, which results in $c \in\left(0,4.5 \times 10^{-3}\right]$. An equation of the form $c(\mu)=a \mu^{b}$ was fitted to the data in Figure 11, and the fitting process reveals $a \in(0.010770,0.011)$ and $b \in(0.416,0.4218)$ with $95 \%$ confidence. In fact, $a=0.01088$ and $b=0.4189$. Literature indicates that the value of $b$ should be higher, with Gilligan [8] and Metz, Mollison and van den Bosch [13] finding the wave speed to be proportional to the square root of the diffusion constant; that is $c \propto \sqrt{\mu}$. Although $b<0.5$ the equation fits the data well, and since $S S E=8.59 \times 10^{-6}$ its use in making predictions would be justified. The coefficient of determination $r^{2}=0.9933$ indicating that $99.33 \%$ of the variance of the data is explained by the equation.

\section{CONCLUSION}

In this work, we have derived a Host-Pathogen model where the PFE is always LAS and may co- exist with endemic equilibria. We provided sufficient conditions for PFE being globally asymptotically stable and for persistence of the pathogen, using two different approaches, LaSalle Invariance Principle approach and monotone system approach. We show that these results can be extended to the spatio-temporal system, where we add diffusion in the free pathogen compartment. We also show numerically that a bi-stable travelling wave solution can exist between PFE and a stable endemic equilibrium, here $\mathcal{E} \mathcal{E}_{2}$. We show that the speed $c$ of this traveling wave is of the form $a \mu^{b}$, where $\mu$ is the diffusion parameter. Further theoretical investigations are needed, in order to be able to derive appropriate control strategies to avoid invasion of the pathogen in the whole crop.

\section{REFERENCES}

[1] R. Anguelov, Y. Dumont, J. Lubuma (2012), Mathematical modelling of sterile insect technology for control of anopheles mosquito, Computers \& Mathematics with 
Applications 64(3), 374-389.

https://doi.org/10.1016/j.camwa.2012.02.068

[2] R. Anguelov, Y. Dumont, and J. M.-S. Lubuma (2012). On nonstandard finite difference schemes in biosciences, AIP Conf. Proc. 1487(1), 212-223. https://doi.org/10.1063/1.4758961

[3] D.P. Bebber, T. Holmes, S.J. Gurr (2014), The global spread of crop pests and pathogens, Glob. Ecol. Biogeogr 23(12), 1398-1407. https://doi.org/10.1111/geb.12214

[4] A. Berman, R.J. Plemmons, Nonnegative matrices in the mathematical sciences, SIAM, 1994.

[5] N.J. Cunniffe (2007), Dispersal of soil-borne plant pathogens and efficacy of biological control, $\mathrm{PhD}$ Thesis, University of Cambridge.

[6] C.A. Gilligan (1985), Construction of temporal models: III. Disease progress of soil-borne pathogens. In: C.A. Gilligan (ed.), Mathematical modelling of crop disease, London, Academic Press.

[7] C.A. Gilligan (1990), Mathematical modeling and analysis of soilborne pathogens. In: J. Kranz (ed.) Epidemics of plant diseases: Mathematical analysis and modeling, Heidelberg, Springer-Verlag, 96-142.

[8] C.A. Gilligan (1995), Modelling soil-borne plant pathogens: reaction-diffusion models, Canadian Journal of Plant Pathology 17, 96-108.

[9] E.E. Holmes, M.A. Lewis, J.E. Banks, R.R. Veit (1994), Partial differential equations in ecology: spatial interac-
Partial differential equations in ecology: spatial interactions and population dynamics, Ecology 75, 17-29.

[10] State of the World's Plants Report 2016, Royal Botanic Gardens, Kew, https://stateoftheworldsplants.org/2016/

[11] J. LaSalle (1960), Some Extensions of Liapunov's Second Method, IRE Transactions on Circuit Theory 7(4), 520-527.

[12] J.D. MacDonald, The Soil Environment. In: C.L. Campbell, D.M. Benson (eds), Epidemiology and Management of Root Disease, Springer-Verlag, 1994, 82-116.

[13] J.A.J. Metz, D. Mollison, F. van den Bosch (2000), The Dynamics of Invasion Waves. In: U. Dieckmann, R. Law, J.A.J Metz (eds), The Geometry of Ecological Interactions: Simplifying Spatial Complexity, Cambridge University Press, 482-512, https://doi.org/10.1017/CBO9780511525537.027

[14] E.C. Oerke (2006). Crop losses to pests, J.Agric. Sci. 144(1), 31-43.

[15] A. Okubo, S.A. Levin, Diffusion and Ecological Problems: Modern Perspectives, Springer, 2001.

[16] H.L. Smith, Monotone Dynamical System, AMS, 1995.

[17] P. van den Driessche, J. Watmough (2002), Reproduction numbers and sub-threshold endemic equilibria for compartmental models of disease transmission, Mathematical Biosciences 180, 29-48, https://doi.org/10.1016/S0025-5564(02)00108-6

[18] W. Walter, Differential and Integral Inequalities, Springer, 1970. 